\title{
The multistage and ring-apodized vortex coronagraph: two simple, small-angle coronagraphic solutions for heavily obscured apertures
}

Dimitri Mawet, Laurent Pueyo, Alexis Carlotti, Bertrand Mennesson, Eugene Serabyn, et al.

Dimitri Mawet, Laurent Pueyo, Alexis Carlotti, Bertrand Mennesson, Eugene Serabyn, James Wallace, Pierre Baudoz, "The multistage and ringapodized vortex coronagraph: two simple, small-angle coronagraphic solutions for heavily obscured apertures," Proc. SPIE 8864, Techniques and Instrumentation for Detection of Exoplanets VI, 886411 (26 September 2013); doi: $10.1117 / 12.2025574$

SPIE Event: SPIE Optical Engineering + Applications, 2013, San Diego, California, United States 


\title{
The multistage and ring-apodized vortex coronagraph: two simple, small-angle coronagraphic solutions for heavily obscured apertures
}

\author{
Dimitri Mawet ${ }^{a, b}$, Laurent Pueyo ${ }^{c}$, Alexis Carlottid ${ }^{d, e}$, Bertrand Mennesson ${ }^{b}$, Eugene Serabyn ${ }^{b}$, \\ James Wallace $^{b}$, and Pierre Baudoz $f$ \\ ${ }^{a}$ European Southern Observatory, Alonso de Córdova 3107, Vitacura, Casilla 19001, Chile; \\ ${ }^{b}$ Jet Propulsion Laboratory, California Institute of Technology, Pasadena, CA 91109, USA; \\ ${ }^{c}$ Space Telescope Science Institute, 3700 San Martin Drive, Baltimore, MD 21218, USA; \\ ${ }^{d}$ Mechanical \& Aerospace Engineering, Princeton University, Olden street, 08544, Princeton, \\ NJ, USA; \\ ${ }^{e}$ Institut de Planétologie et d'Astrophysique de Grenoble (IPAG), University Joseph Fourier, \\ CNRS, BP 53, 38041, Grenoble, France;

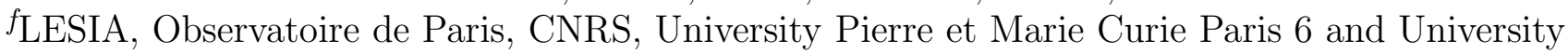 \\ Denis Diderot Paris 7, 5 place Jules Janssen, 92195 Meudon, France
}

\begin{abstract}
Here we present two simple concepts to make the vortex coronagraph (VC) immune to heavily obscured apertures. The multi-stage VC (MSVC) uses the ability of the vortex to move light in and out of apertures through multiple $\mathrm{VC}$ in series to restore the nominal attenuation capability of the charge 2 vortex regardless of the aperture obscurations. The ring-apodized VC (RAVC) is a one-stage apodizer exploiting the VC Lyot-plane amplitude distribution in order to perfectly null the diffraction from any central obscuration size, and for any vortex topological charge. In this paper, we also emphasize the complementarity and similarities of the RAVC to the recently proposed Active Compensation of Aperture Discontinuities (ACAD, L. Pueyo et al. 2013, these proceedings) and more finely optimized shaped-pupil-like apodizations (A. Carlotti et al. 2013, these proceedings). This paper ends with a brief discussion about the trade-offs these techniques offer in the framework of the Extremely Large Telescopes (ELT) and the Astrophysics Focused Telescope Assets (AFTA).
\end{abstract}

Keywords: High contrast imaging, vortex coronagraphy, on-axis telescopes, apodization, AFTA telescope, Extremely Large Telescopes

\section{INTRODUCTION}

The vortex coronagraph (VC, see, e.g. Ref. 1) is one of the most advanced coronagraphs recently brought to operational level. ${ }^{2,3}$ The VC offers small inner working angle (IWA), potentially down to the diffraction limit $(0.9 \lambda / D)$, clear $360^{\circ}$ off-axis field of view/discovery space, unlimited outer working angle, high throughput, intrinsic and/or induced achromaticity, operational simplicity, and compatibility with the Lyot coronagraph layout. It has also recently demonstrated $\simeq 10^{-9}$ raw contrast levels in the visible on the High Contrast Imaging Testbed (HCIT) at the Jet Propulsion Laboratory (see E. Serabyn et al. 2013, these proceedings, and Ref. 3). It is also equipping state of the art high-contrast instruments of various 5-8 $m$ class telescopes. Since it opens a new parameter space at small separations, it has enabled recent scientific results at Palomar in the $\mathrm{H}$ and $\mathrm{K}$ bands, ${ }^{4-7}$ and at the Very Large Telescope ${ }^{8}$ in the L' band (see also O. Absil et al. 2013, in preparation, and J. Milli et al. 2013, in preparation). It is currently being implemented on SCExAO at Subaru ${ }^{9}$ and on LMIRCAM at the Large Binocular Telescope. ${ }^{10,11}$ It is also a candidate coronagraph for an exoplanet characterization space-based mission (WFIRST-AFTA, see Ref. 12,13; ACCESS, see Ref. 14; and SPICES, see Ref. 15), for the EuropeanExtremely Large Telescope, ${ }^{3}$ and the Thirty-Meter Telescope.

Further author information: send correspondence to dmawet@eso.org

Techniques and Instrumentation for Detection of Exoplanets VI, edited by Stuart Shaklan, Proc. of SPIE Vol. 8864, 886411 · C 2013 SPIE · CCC code: 0277-786X/13/\$18 · doi: 10.1117/12.2025574 
However, as for all other coronagraphs, the $\mathrm{VC}$ is sensitive to the aperture geometry, and particularly to secondary obscurations. ${ }^{16,17}$ This sensitivity stems from the fact that a vortex phase ramp in the focal plane of a telescope always diffracts light to the outer regions of circularly symmetric pupil intensity discontinuities. Thus, as expected, a single vortex will move light outside of the secondary obscuration and support structures, right into the primary pupil image (Fig. 1, A). The subsequent contrast degradation is proportional to the obscured area $\left(r_{0} / R\right)^{2}$, with $r_{0}$ and $R$ being the radii of the central obscuration and primary mirror, respectively. ${ }^{16}$

Recently we proposed a method, ${ }^{17}$ based on multiple vortices, that, without sacrificing throughput, reduces this residual light leakage to $\left(r_{0} / R\right)^{2 n}$, with $n$ the number of coronagraph stages. This method thus enables high contrasts to be reached even with an on-axis telescope, but at the cost of increased optical complexity, and for an imperfect result. Here we propose two new solutions to this problem that now render the VC completely insensitive to central obscurations.

\section{POSITION OF THE PROBLEM}

A vortex coronagraph is a transparent mask which multiplies the telescope point spread function by a helical phase of the form $e^{i \phi}$, with $\phi=l \theta$, where $\theta$ is the focal plane azimuthal coordinate and $l$ the (even) vortex "topological charge". The light then propagates downstream to the "Lyot" plane, where for an ideal unobscured circular input pupil, all of the (on-axis) starlight appears outside of the geometric image of the input pupil. A so-called Lyot stop then blocks the diffracted starlight. Light from off-axis objects misses the center of the vortex and propagates normally.

The detailed effect of a charge $l=2$ vortex phase ramp, $e^{i 2 \theta}$, applied to the ideal focal plane field (Airy pattern), $\frac{2 J_{1}(k \rho R)}{k \rho R}$, of a filled circular aperture of radius $R$, where $\mathrm{k}$ is the wavenumber and $\rho$ is the radial coordinate in the focal plane, has been calculated analytically by various authors. ${ }^{1,18-20}$ The Fourier transform of focal plane electrical field $e^{i 2 \theta} \frac{2 J_{1}(k \rho R)}{k \rho R}$ gives the field in the pupil plane downstream from the coronagraph (Lyot-stop plane). Dropping the azimuthal phase term, this transform yields:

$$
E_{L}(r)= \begin{cases}0 & r<R \\ \left(\frac{R}{r}\right)^{2} & r>R\end{cases}
$$

Using the superposition principle, a centrally obscured pupil can be seen as the difference between a filled pupil of radius $R$ and a smaller filled pupil of radius $r_{0}$, yielding a pupil field after the topological charge 2 vortex of $^{17}$

$$
E_{L}(r)= \begin{cases}0 & r<r_{0} \\ -\left(\frac{r_{0}}{r}\right)^{2} & r_{0}<r<R \\ {\left[\left(\frac{R}{r}\right)^{2}-\left(\frac{r_{0}}{r}\right)^{2}\right]} & r>R\end{cases}
$$

The Lyot stop then blocks everything for $r>R$, so from now on, we will not consider this area anymore in order to focus on the region of interest, i.e. $r<R$. Indeed, the residual field interior to the pupil (between $r_{0}$ and $R$ ) leads to contrast degradation in the subsequent focal plane image, as the fraction of the total energy remaining inside the pupil is $\left(r_{0} / R\right)^{2}$, or 0.04 for a $20 \%$ central obscuration.

\section{MULTI-STAGE VORTEX CORONAGRAPH (MSVC)}

Our new multi-stage approach starts with the double-stage vortex coronagraph concept presented in Ref. 17 and demonstrated in the laboratory by Serabyn et al. 2013 (in preparation), but will depart from it to cancel the residual light leakage from $\left(r_{0} / R\right)^{2 n}$, with $n$ the number of coronagraph stages, to basically, 0 . 

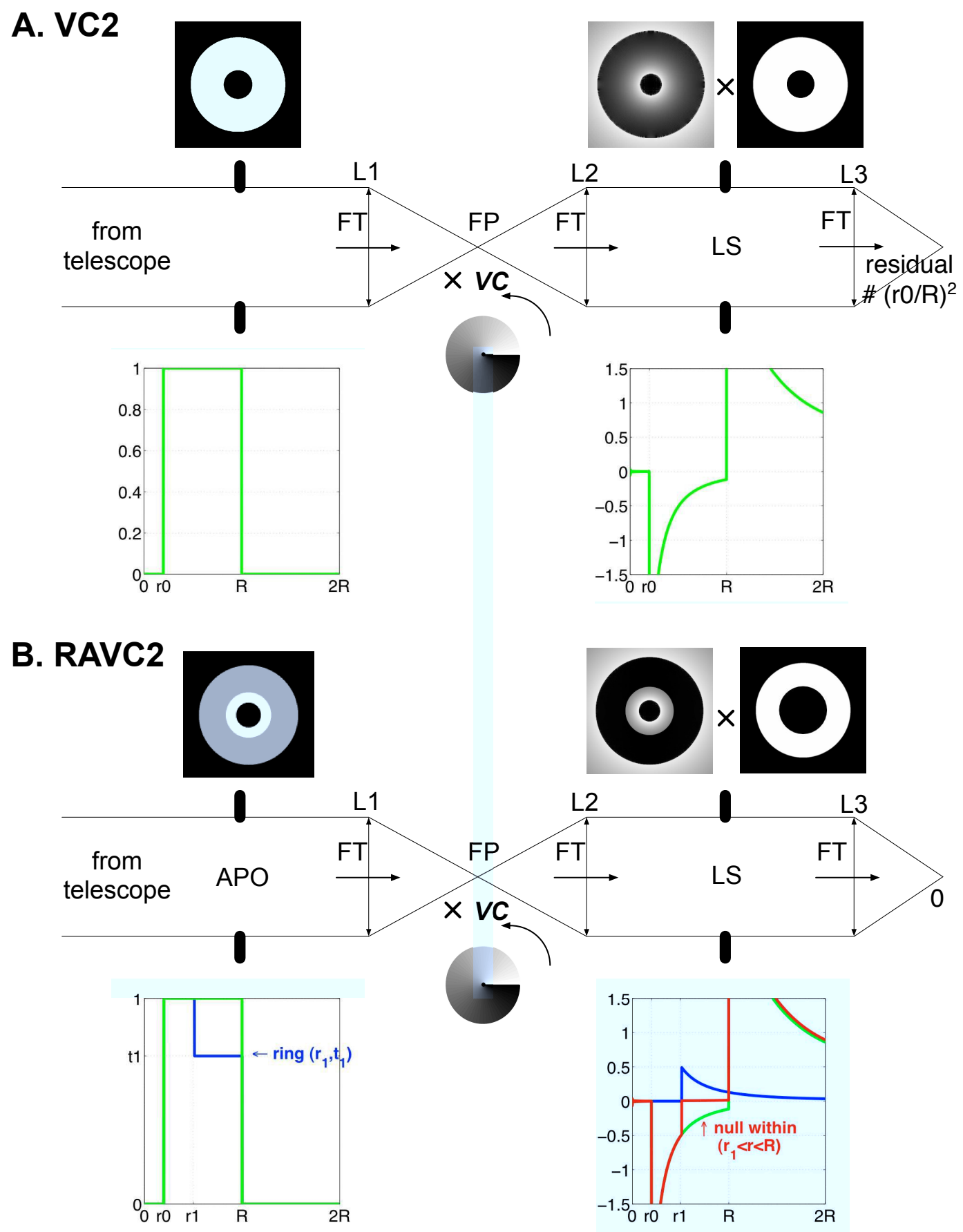

Figure 1. A: classical VC of topological charge 2 with a centrally obscured telescope of radius $R$ ( $r_{0}$ is the radius of the secondary shadow). The residual field interior to the pupil (between $r_{0}$ and $R$ ) leads to contrast degradation in the subsequent focal plane image, as the fraction of the total energy remaining inside the pupil is $\left(r_{0} / R\right)^{2}$, or 0.04 for a $20 \%$ central obscuration. B.: RAVC of topological charge 2 . The ring of radius $r_{1}$ and amplitude transmittance $t_{1}$ is optimized so that the overlap of the self-similar vortex functions at the Lyot plane issued from the central obscuration (green curve) and the ring (blue curve) perfectly cancel each other between $r_{1}$ and $R$ (red curve).

\subsection{Principle}

Following Eq. 2 presented above, reintroducing the $e^{i 2 \psi}$ vortex phase tern, using the superposition principle and the self-similarity of the $1 / r^{2}$ terms, the field after the first Lyot stop can be rewritten (Fig. $2, \mathbf{C}^{+}$) 


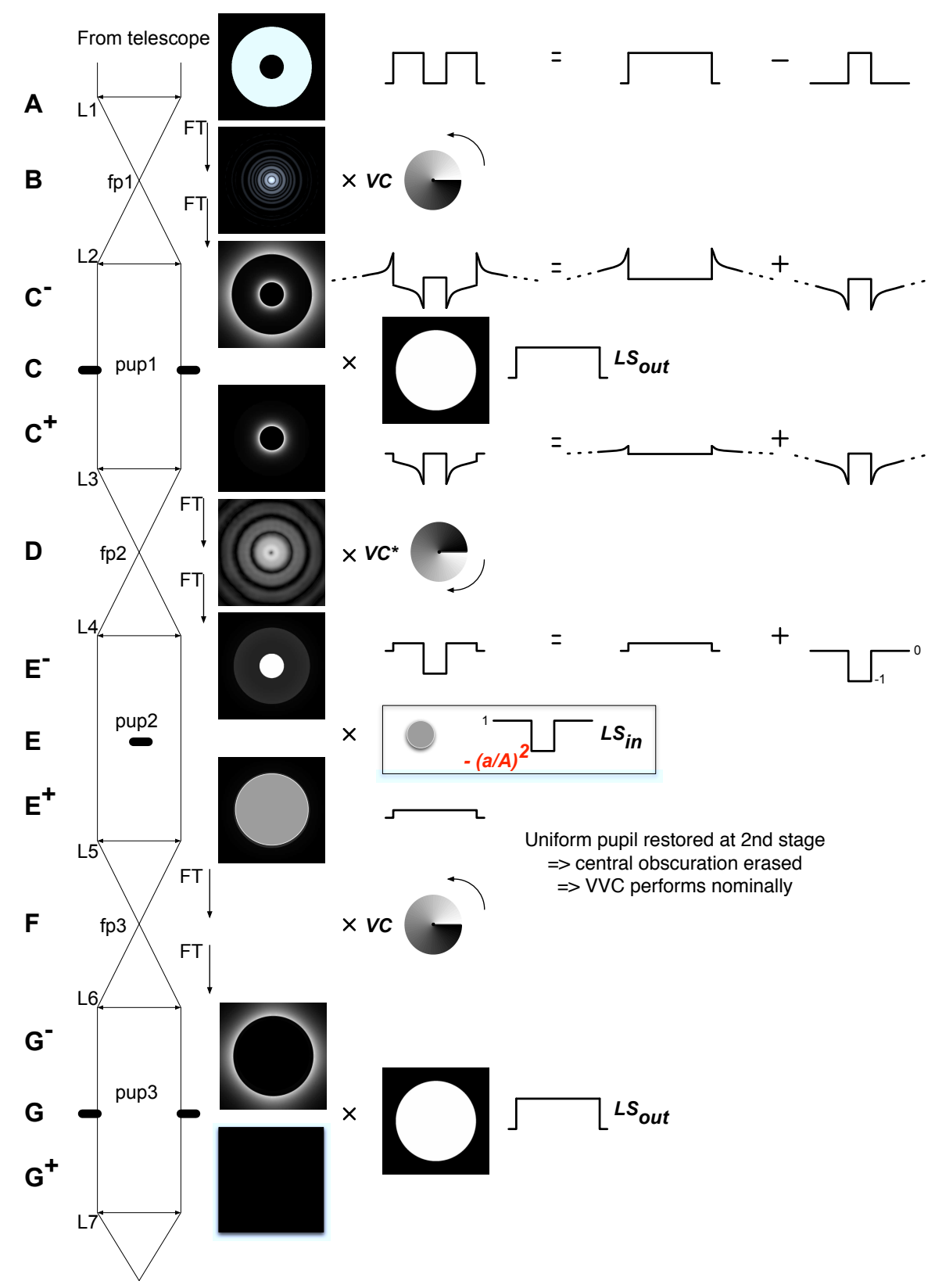

Figure 2. Layout of the multi-stage vortex coronagraph. A. Obscured entrance pupil seen as the difference of two filled apertures with radii $R$ and $r_{0}<R$. B. First Vortex Coronagraph $(V C)$ phase ramp $e^{i \phi}$ applied to the image produced by the imaging lens L1. $\mathbf{C}^{-}$. The pupil plane distribution after pupil imaging lens L2. The crosscuts show the linear decomposition of the field into the sum of a positive term $(R / r)^{2}$ for the filled pupil of radius $R$, and another negative term $-\left(r_{0} / r\right)^{2}$ corresponding to the filled pupil of radius $r_{0}<R$. C. First Lyot stop $L S_{\text {out }}$. $\mathbf{C}^{+}$. Post Lyot stop field. The crosscuts show the decomposition of the residual field as the sum of the negative pupil term $-\left(r_{0} / r\right)^{2}$, and a second term that leads to cancellation beyond $R$. D. Image in the second focal plane after L3, multiplied by the conjugated vortex $V C^{*}$, with a phase ramp $e^{-i \phi} . \mathbf{E}^{-}$. Pupil plane field after the second vortex, decomposed into the sum of an outer pupil of radius $R$ with a reduced amplitude $\left(r_{0} / R\right)^{2}$, and the inner pupil of radius $r_{0}$ of amplitude -1 . E. Application of the second semi-transparent inner Lyot stop $\left(L S_{i n}\right) \cdot \mathbf{E}^{+}$. The exit pupil after the second stage is now uniform with an intensity reduced by the factor $\left(r_{0} / R\right)^{4}$. The third stage $\left(\mathbf{F}, \mathbf{G}^{-}, \mathbf{G}, \mathbf{G}^{+}\right)$now acts as a perfect nominal vortex.

$$
E_{\text {Lyot } 1}(r, \psi)= \begin{cases}0 & r<r_{0} \\ -e^{i 2 \psi}\left(\frac{r_{0}}{r}\right)^{2} & r_{0}<r<R \\ e^{i 2 \psi}\left(\frac{r_{0}}{R}\right)^{2}\left(\frac{R}{r}\right)^{2}-e^{i 2 \psi}\left(\frac{r_{0}}{r}\right)^{2} & r>R\end{cases}
$$


We now go to a second stage focal plane by Fourier transforming Eq. 3, yielding

$$
E_{f p 2}(\rho, \theta)=-e^{i 2 \theta} \frac{2 J_{1}\left(k \rho r_{0}\right)}{k \rho r_{0}}+e^{i 2 \theta} \frac{2 J_{1}(k \rho R)}{k \rho R}\left(\frac{r_{0}}{R}\right)^{2}
$$

Multiplying this function by the conjugated vortex $e^{-i 2 \theta}$ cancels the phase term (Fig. 2, D), leaving

$$
E_{f p 2}(\rho, \theta)=-\frac{2 J_{1}\left(k \rho r_{0}\right)}{k \rho r_{0}}+\frac{2 J_{1}(k \rho R)}{k \rho r_{0}}\left(\frac{r_{0}}{R}\right)^{2}
$$

Transforming back to a second pupil plane we get (Fig. 2, $\mathbf{E}^{-}$)

$$
E_{\text {pup } 2}(r, \psi)=\left\{\begin{array}{ll}
-1 & r<r_{0} \\
0 & r>r_{0}
\end{array}\right\}+\left\{\begin{array}{ll}
\left(\frac{r_{0}}{R}\right)^{2} & r<R \\
0 & r>R
\end{array}\right\}
$$

Now, the following departs from the double-stage concept exposed in Ref. 17. Indeed instead of blocking the bright central region by a second fully opaque Lyot stop (Fig. 2, E), of radius $r_{0}$, which leaves, we use a phaseshifter semi-transmissive Lyot stop whose goal is to make the output pupil of stage 2 uniform in phase and amplitude

$$
E_{\text {Lyot } 2}(r, \psi)= \begin{cases}\left(\frac{r_{0}}{R}\right)^{2} & r<r_{0} \\ \left(\frac{r_{0}}{R}\right)^{2} & r_{0}<r<R \\ 0 & r>R\end{cases}
$$

The Lyot stop at stage 2 thus does the following

$$
\operatorname{Lyot} 2(r, \psi)= \begin{cases}-\left(\frac{r_{0}}{R}\right)^{2} /\left(1-\left(\frac{r_{0}}{R}\right)^{2}\right) & r<r_{0} \\ 1 & r_{0}<r<R \\ 0 & r>R\end{cases}
$$

The output of stage 2 now is send to a third VVC which acts nominally to provide the expected perfect rejection thanks to the use of a simple third Lyot stop identical to the first one.

$$
E_{\text {Lyot } 3}(r, \psi)= \begin{cases}0 & r<R \\ e^{i 2 \psi}\left(\frac{r_{0}}{R}\right)^{2}\left(\frac{R}{r_{0}}\right)^{2} & r>R\end{cases}
$$

\subsection{Practical considerations}

The obvious main feature of the advanced multi-stage concept is the lossless perfect immunization to any central obscuration. Moreover, multi-stage coronagraphy has also been proposed previously as a way to increase the working bandwidth and reduce the sensitivity to tip-tilt and stellar size leakage ${ }^{21-23}$ see Fig. 3 (right). Note that the inner working angle of three topological charge $2 \mathrm{VCs}$ in cascade is $1.75 \lambda / d$ (see Fig. 3, left).

\section{PRINCIPLE OF THE RAVC}

While the multi-stager is interesting from a theoretical standpoint, its practical implementation requires many optics, which imposes system-level constraints not to be underestimated. So we now present a simpler concept. The ring-apodized vortex coronagraph (RAVC) is based on the superposition principle and the vortex properties of moving light in and out of circular apertures. Its principle relies on modulating the entrance pupil with one (or a set of) concentric ring(s) of well chosen size(s) and transmittance(s), in order to yield perfect cancellation of on-axis sources at the Lyot stop level. In the following, we show that perfect solutions can be found for any topological charge. We will start with the case of topological charge $2 \mathrm{RAVC}$, and detail the derivation for charge 4 RAVC in the next section. 

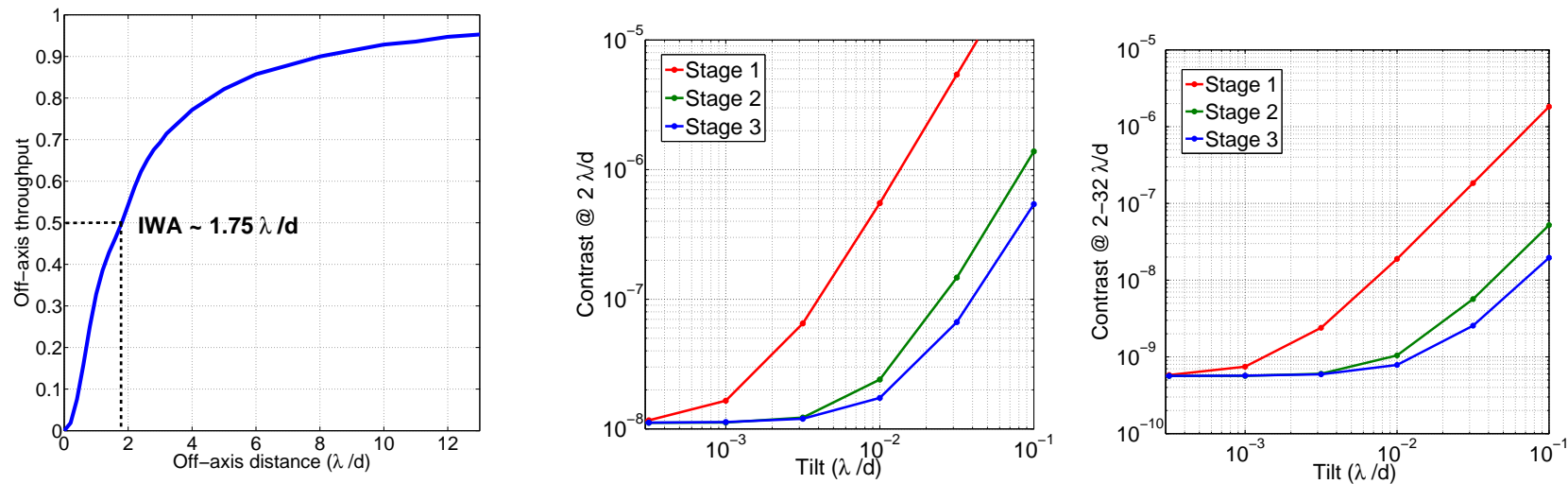

Figure 3. Left: off-axis throughput, and IWA of the 3-stage MSVC. Middle: sensitivity to tip-tilt at $2 \lambda / d$. Right: sensitivity to tip-tilt between 2 and $32 \lambda / d$.

\subsubsection{One Ring to rule the diffraction, One Ring to null it, and in the darkness...}

Consider now that the entrance pupil has an additional ring with $r$ from $r_{1}$, such that $r_{0}<r_{1}<R$, to the outer radius $R$, and characterized by an amplitude transmission coefficient $t_{1}$ for $r_{1}<r<R$. Note that the interior of the ring, $r_{0}<r<r_{1}$, has a transmission $t_{0}=1$. Using the same reasoning, we now have within the Lyot plane after the charge 2 vortex

$$
E_{L}(r)= \begin{cases}0 & r<r_{0} \\ -\left(\frac{r_{0}}{r}\right)^{2} & r_{0}<r<r_{1} \\ \left(1-t_{1}\right)\left(\frac{r_{1}}{r}\right)^{2}-\left(\frac{r_{0}}{r}\right)^{2} & r_{1}<r<R\end{cases}
$$

It appears clearly that the degrees of freedom introduced by the ring apodizer (namely its size $r_{1}$, and transmittance $t_{1}$ ) provide enough leverage to completely cancel the light within $r_{1}<r<R$. Indeed if

$$
\left(1-t_{1}\right)=\left(\frac{r_{0}}{r_{1}}\right)^{2}
$$

then the field in the Lyot plane for $r_{1}<r<R$ is completely nulled. Fig. 1, B shows 1-dimensional calculations and 2-dimensional simulations where, as expected, the vortex fields issued from the central obscuration and the ring perfectly balance and cancel each other at the Lyot plane, between $r_{1}$ and $R$ downstream from the VC. The single Lyot stop is then designed to block the light for $0<r<r_{1}$, thus effectively increasing the size of the final central obstruction, and of course for $r>R$.

\subsubsection{Throughput optimization}

There is a whole set of solutions to Eq. 11 with $0<t_{1}<1$ and $r_{0}<r_{1}<R$. However, the best solution will maximize the throughput $T$ for a given $r_{0}$. $T$ is defined as the energy going through the $\operatorname{ring} r_{1}<r<R$, normalized by the energy nominally transmitted by the centrally obscured telescope aperture, or

$$
T=\frac{t_{1}^{2}\left(1-\left(\frac{r_{1}}{R}\right)^{2}\right)}{1-\left(\frac{r_{0}}{R}\right)^{2}}
$$

Substituting Eq. 11 into Eq. 12, and differentiating $\mathrm{T}$ with respect to $t_{1}$, we find the optimal ring parameters associated with a charge $2 \mathrm{VC}$ 


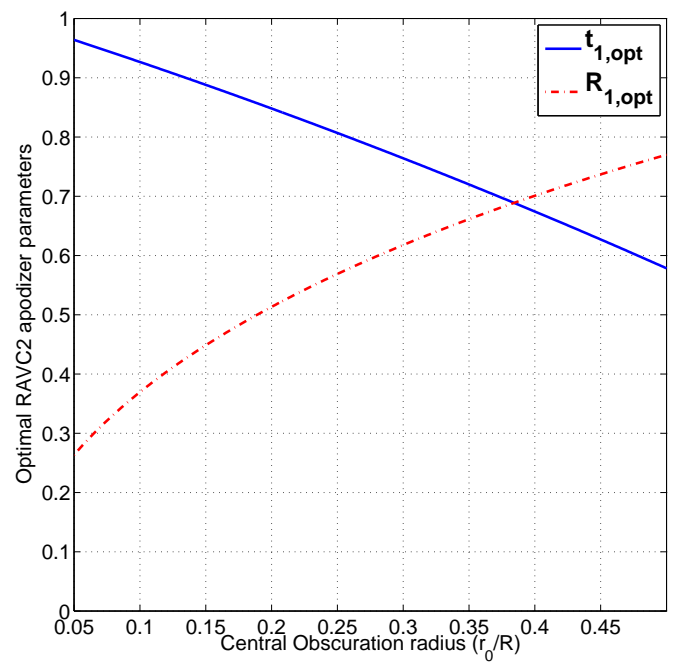

Figure 4. Optimal apodizer parameters for a charge 2 ring-apodized vortex coronagraph (RAVC2), $t_{1, \text { opt }}$ and $R_{1, \text { opt }}$, as a function of $r_{0} / R$.

$$
\left\{\begin{array}{l}
t_{1, o p t}=1-\frac{1}{4}\left(R_{0}^{2}+R_{0} \sqrt{R_{0}^{2}+8}\right) \\
R_{1, o p t}=\frac{R_{0}}{\sqrt{1-t_{1, o p t}}}
\end{array}\right.
$$

where $R_{0}=r_{0} / R$, and $R_{1}=r_{1} / R$ are the relative radii. Note that $t_{1, o p t}$ and $R_{1, \text { opt }}$ are functions of $r_{0} / R$ only (see Fig. 4), which is remarkably analogous to the problem associated to designing apodizers for apodized pupil Lyot coronagraphs (APLC) with hard edge focal plane masks. ${ }^{24}$ Indeed, in both cases, there only exists a unique apodizer configuration that maximizes throughput while yielding a chosen level of starlight extinction. However due to the nature of the VC, this optimal solution turns out to rely on sharp variation of the amplitude profile while the optimal solutions for an APLC are smooth.

\subsection{Charge 4 RAVC}

The charge 2 RAVC design is simple and the analytical solution very easy to find. The cancellation of the field at the Lyot stop within the outer ring, and the throughput maximization provides two equations that fully and unambiguously characterize the apodizer's two free parameters. The charge 4 case is similar in nature but slightly less trivial.

\subsubsection{Two rings for perfect cancellation}

As the topological charge of the VC increases, so does the complexity of its response at the Lyot stop plane. Following Ref. 1 and Ref. 20, for a topological charge 4 vortex, we have

$$
E_{L}(r)= \begin{cases}0 & r<R \\ 2\left(\frac{R}{r}\right)^{2}-3\left(\frac{R}{r}\right)^{4} & r>R\end{cases}
$$

The amplitude function after the vortex is now a polynomial of order -4 , following the topological charge $l$ of the vortex. This function is not self-similar anymore, even though each individual term is. For the sake of simplicity, let us rename this polynomial

$$
V_{4}(r, R)=2\left(\frac{R}{r}\right)^{2}-3\left(\frac{R}{r}\right)^{4}
$$


A single additional ring will not provide enough leverage to cancel both terms, so we will now consider adding a second ring with $r$ from $r_{2}$, such that $r_{1}<r_{2}<R$, to the radius $R$, and characterized by an amplitude transmission coefficient $t_{2}$ for $r_{2}<r<R$. Note that the first ring is now of inner radius $r_{1}$, such that $r_{0}<r_{1}<R$ and outer radius $r_{2}$, and characterized by an amplitude transmission coefficient $t_{1}$ for $r_{1}<r<r_{2}$. Note that the interior of the first ring, $r_{0}<r<r_{1}$, still has a transmission $t_{0}=1$.

Using the same reasoning as before, we now have within the Lyot plane after the charge 4 vortex, and this double ring apodizer

$$
E_{L}(r)= \begin{cases}0 & r<r_{0} \\ -V_{4}\left(r, r_{0}\right) & r_{0}<r<r_{1} \\ \left(1-t_{1}\right) V_{4}\left(r, r_{1}\right)-V_{4}\left(r, r_{0}\right) & r_{1}<r<r_{2} \\ \left(t_{1}-t_{2}\right) V_{4}\left(r, r_{2}\right)+ & \\ \left(1-t_{1}\right) V_{4}\left(r, r_{1}\right)-V_{4}\left(r, r_{0}\right) & r_{2}<r<R\end{cases}
$$

We are now seeking solutions that perfectly cancel the light within the outer ring $r_{2}<r<R$, using the four free parameters constraining the ring sizes and transmittances, i.e. $r_{1}, r_{2}$ and $t_{1}, t_{2}$.

$$
\left(t_{1}-t_{2}\right) V_{4}\left(r, r_{2}\right)+\left(1-t_{1}\right) V_{4}\left(r, r_{1}\right)-V_{4}\left(r, r_{0}\right)=0
$$

Finding solutions to this under-constrained problem is not straightforward as the $V_{4}(r, R)$ functions are not self similar. However, by separating the quadratic and fourth order terms, and, since $r>0$, we can rewrite Eq. 17 as

$$
\left\{\begin{array}{l}
\left(t_{1}-t_{2}\right)\left(r_{2}\right)^{2}+\left(1-t_{1}\right)\left(r_{1}\right)^{2}-\left(r_{0}\right)^{2}=0 \\
\left(t_{1}-t_{2}\right)\left(r_{2}\right)^{4}+\left(1-t_{1}\right)\left(r_{1}\right)^{4}-\left(r_{0}\right)^{4}=0
\end{array}\right.
$$

Eq. 18 is a system of two equations with 4 unknowns.

\subsubsection{Throughput optimization}

In order to better constrain the set of possible solutions, we once again introduce throughput as our figure of merit, but now $T$ is defined as the energy going through the ring $r_{2}<r<R$.

$$
T=\frac{t_{2}^{2}\left(1-\left(\frac{r_{2}}{R}\right)^{2}\right)}{1-\left(\frac{r_{0}}{R}\right)^{2}}
$$

Closer examination of the throughput expression indicates that optimal solutions are solutions that maximize the outer ring transmittance $t_{2}$ while keeping $r_{2}$ as small as possible. Cascading both constraints down to Eq. 18, it is easy to derive that such a condition is met for $t_{1}=0$. Indeed, with $t_{1}=0$, the modulation terms in $r_{1}$

and $r_{2}$ introduced by the rings to balance the central obscuration $r_{0}$ terms, have maximum weights. Setting $t_{1}=0$ allows us to simplify the equations greatly, yielding the following charge 4 ring-apodized $\mathrm{VC}$ fundamental formulae for optimal throughput

$$
\left\{\begin{array}{l}
R_{1}=\sqrt{\sqrt{R_{0}^{2}\left(R_{0}^{2}+4\right)}-2 R_{0}^{2}} \\
R_{2}=\sqrt{R_{1}^{2}+R_{0}^{2}} \\
t_{2}=\frac{R_{1}^{2}-R_{0}^{2}}{R_{1}^{2}+R_{0}^{2}}
\end{array}\right.
$$

where $R_{0}=r_{0} / R, R_{1}=r_{1} / R, R_{2}=r_{2} / R$, are the radii relative to the entrance pupil outer radius $R$. One can also explore the entire parameter space $\left(r_{1}, r_{2}, t_{1}, t_{2}\right)$ by solving numerically the following optimization problem: 


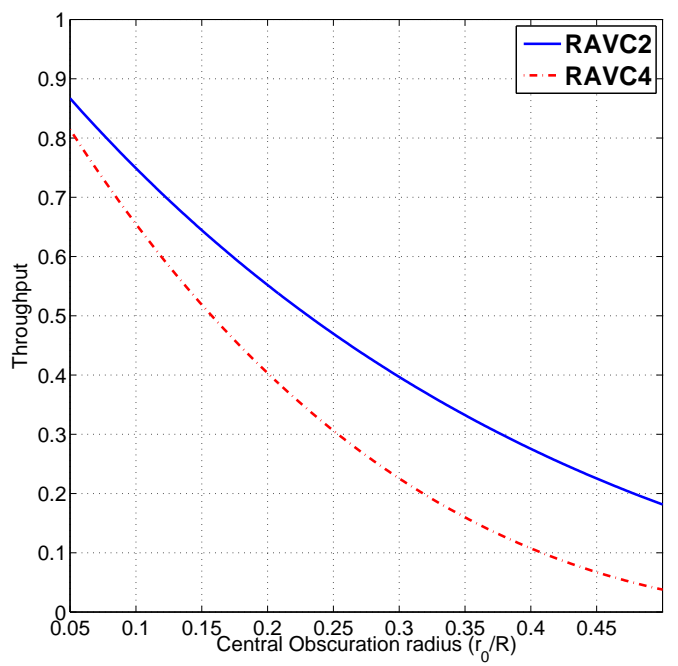

Figure 5. Theoretical maximum throughput of the RAVC2 and RAVC4 with transmissive ring apodizers for various obscuration relative diameters $r_{0} / R$. The throughput decreases with the topological charge and central obscuration.

$$
\begin{aligned}
& \text { Maximize } t_{2}^{2}\left(1-\left(\frac{r_{2}}{R}\right)^{2}\right) \\
& \text { s.t. }\left\{\begin{array}{l}
\left(t_{1}-t_{2}\right)\left(r_{2}\right)^{2}+\left(1-t_{1}\right)\left(r_{1}\right)^{2}-\left(r_{0}\right)^{2}=0 \\
\left(t_{1}-t_{2}\right)\left(r_{2}\right)^{4}+\left(1-t_{1}\right)\left(r_{1}\right)^{4}-\left(r_{0}\right)^{4}=0
\end{array}\right.
\end{aligned}
$$

This optimization naturally yields solutions for which $t_{1}=0$, for all sizes of central obscurations.

\subsection{Performances}

Here we discuss the performance of the RAVC family in terms of contrast, (off-axis) throughput, and inner working angle. For perfect optics and perfect VCs of various (even) topological charges, there exists RAVC solutions providing infinite contrast whatever the central obscuration. Throughput is a decreasing function of the topological charge and central obscuration size. Indeed, throughput is always a function of the outer ring area, which gets smaller when the charge increases (more rings necessary) and of course when the central obscuration gets larger.

Higher topological charges $l$, which trade off inner working angle (e.g. $\mathrm{IWA}_{l=2}=0.9 \lambda / D, \mathrm{IWA}_{l=4}=1.75 \lambda / D$ ), are desired when the telescope size increases (to mitigate the stellar size effect) or when sensitivity to low-order aberrations becomes the limiting factor. ${ }^{16}$ Indeed, Ref. 18 showed that the sensitivity of the VC to pointing offsets $\theta$, in units of $\lambda / D$, is proportional to $\theta^{l}$, with $\theta<<1$ (the same laws apply to the sensitivity to stellar size, which can be seen as an incoherent sum of pointing offsets).

Fig. 5 presents a throughput curve for the RAVC2 and RAVC4, as a function of central obscuration size. For a $10 \%$ central obscuration, the RAVC2 throughput is $\simeq 75 \%$ and $\simeq 65 \%$ for the RAVC4. For $20 \%$, the RAVC2 throughput is $\simeq 55 \%$ and $\simeq 40 \%$ for the RAVC4. Note that the IWA of the VC, classically defined as the $50 \%$ off-axis throughput point (relative to the maximum), is not affected by the apodizer in the topological charge 2 case (see Fig. 6, left), but marginally affected for the charge 4 case (see Fig. 6, right), especially as the size of the central obscuration increases.

The RAVC solution is thus a good compromise between the numerically-optimized apodizer masks presented in Carlotti et al. 2013 (in preparation, see also Section 5.2) as it has comparable throughput but with a full search area, and the Phase-Induced Amplitude Apodization Complex Mask Coronagraph [25, PIAACMC], which involves more complicated optics. 

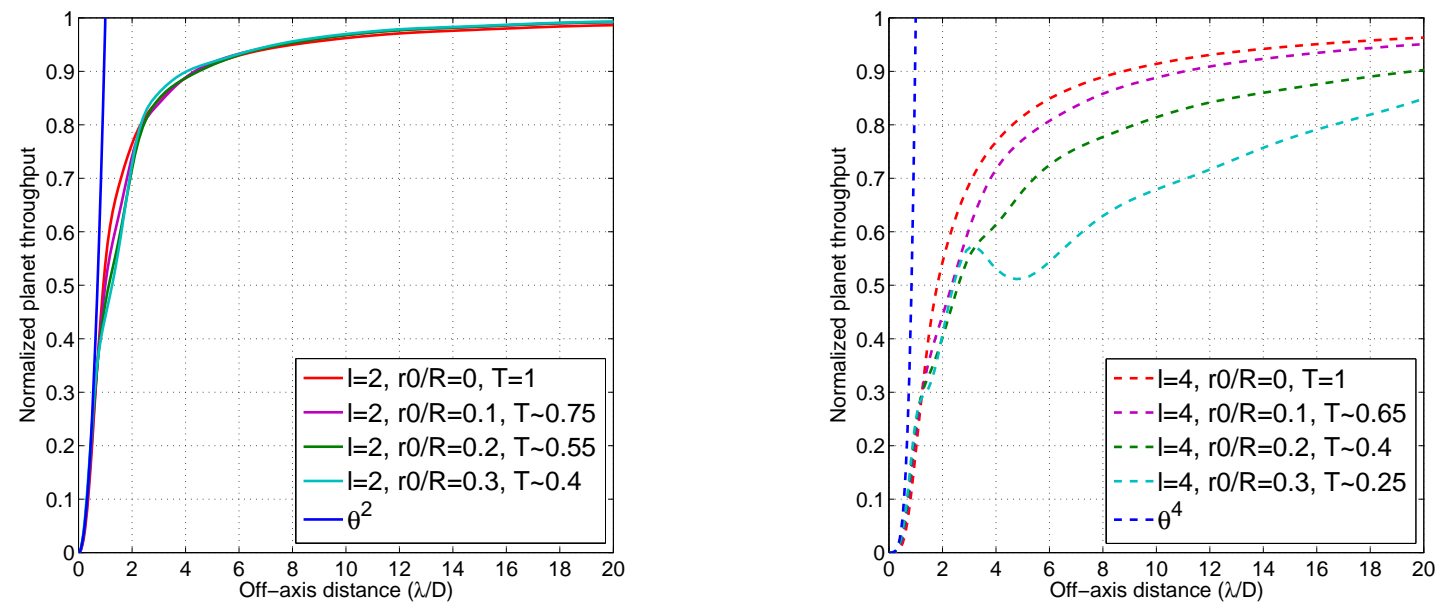

Figure 6. Left: normalized off-axis companion throughput for the RAVC2 (charge $l=2$ ) as a function of angular separation in $\lambda / D$ units. Right: normalized off-axis companion throughput for the RAVC4 (charge $l=4$ ) as a function of angular separation in $\lambda / D$ units. The different curves are for different obscuration ratios. We over-plotted the $\theta^{l}$ function, with the pointing offsets $\theta$ in units of $\lambda / D$, representative of the $\mathrm{VC}$ sensitivity to low-order aberrations (here tip-tilt) for $\theta<<1$.

\subsection{Technical feasibility of the RAVC}

In this section we discuss the technical feasibility of the RAVC, from the current technology readiness of the VC to the ring apodizer manufacturability and the optical layout of the concept, including three practical solutions to mitigate the diffraction from the secondary support structures.

\subsubsection{Vortex mask manufacturing}

The vector vortex coronagraph $[1,26, \mathrm{VVC}]$ is one possible and easy route to manufacture $\mathrm{VCs}^{*}$. It advantageously makes use of the geometrical or Pancharatnam-Berry phase, which is achromatic by nature. The VVC is based on a space-variant halfwave plates (HWP), circularly symmetric in the charge 2 case. Manufacturing the VVC thus requires manipulating the polarization vector in a space-variant manner, i.e. it needs to be significantly modulated across spatial scales of less than a mm, with precisions of a few microns and fractions of a degree. Three technological approaches are currently used to manufacture the VVC: ${ }^{3}$ liquid crystal polymers, ${ }^{26}$ subwavelength gratings, ${ }^{1,28}$ and photonic crystals. ${ }^{29,30}$ Each one of these technological choices has advantages and drawbacks, enumerated in Ref. 3, and practical vortex devices that have already provided very high contrast with unobscured apertures are already available. Thus we now turn to the manufacture of the new component needed, i.e., the ring apodizer.

\subsubsection{Apodizing Mask manufacturing}

Given the extreme simplicity of the ring apodized masks, and their discrete levels of transmittance, no difficulty is foreseen in this area. The manufacturing of the ring apodizer pupil mask should thus be straightforward and one can envision using either microdot or optical coating technologies.

The microdot technology uses a halftone-dot process, where the relative density of a binary array of pixels (transmission of 0 or 1 at the micron level) is calculated to obtain the required local transmission (here uniform within the rings). The manufacturing of current apodized pupil Lyot coronagraphs ${ }^{24,31}$ for SPHERE ${ }^{32}$ and GPI ${ }^{33}$ uses the microdot technology which is well mastered. ${ }^{34,35}$ Note the band-limited coronagraphs of NIRCAM soon to fly aboard JWST have also made use of a similar technique. ${ }^{36}$ The demonstrated advantages of microdot

${ }^{*}$ Noteworthy progress was recently made in the scalar vortex technology, here using computer generated holograms, see Ref. 27. 
apodizers are numerous: 1\%-level accuracy on the transmission profile, achromatic in phase and amplitude, compatibility with a wide range of substrate material, and with conventional AR coating. Spatial phase distortion are in principle absent, ${ }^{34,35}$ but careful control will be necessary for the RAVC. Indeed, the perfect superposition of the fields originating from the central obscuration and the ring(s) requires a uniform phase across the apodizer area.

Another potential technique could make use of optical coatings. Ref. 37 developed a successful method to induce a quasi-achromatic spatially variable optical density with a combination of a deposited metal together with a dielectric to cancel the induced phase shift. This technology has been used to manufacture the bandlimited coronagraph currently holder of the contrast world record, ${ }^{38}$ and spatial transitions of the order of a few microns should be possible.

\subsubsection{RAVC Layout}

The RAVC layout is quite simple and only requires the insertion of the apodizer at a pupil plane upstream of the coronagraph (see Fig. 1, B). Provided that the pupil plane can be shared with a potential deformable mirror, or that the deformable mirror can be slightly out of the pupil plane, no additional stage is required. ${ }^{17}$ Such a configuration allows implementing the RAVC on existing ground-based instruments with little additional effort as wheels with pupil masks are most of the time available.

\section{STRUT MITIGATION}

The analytical solutions presented above only deals with a central on-axis obscuration. Large telescope apertures rarely resemble uniform disks, or annuli. Besides the central obscuration, they usually feature opaque areas produced for example by their support structures or gaps between main mirror segments. Following the superposition principle, such opaque areas diffract light in the same way but with opposite phase. The resulting PSF structure produced by opaque areas can be detrimental to high-contrast imaging, and the total scattered flux is proportional to the size of the obscured area.

Secondary mirror spiders produce extended spike-shaped features, and the net-like gap structure of a segmented mirror produces a regular speckle pattern with a pitch that is inversely proportional to the segment pitch. An efficient diffraction control system has to take aperture irregularities into account. Conventional Apodizers have been calculated for irregular apertures ${ }^{39}$ and are now optimized to deal with phase mask coronagraphs see Sect. 5.2 below, and Ref. 40 .

\subsection{Strut mitigation with ACAD}

An interesting alternative to classical apodization techniques is the upfront correction of aperture irregularities by optical remapping in the geometric and thus achromatic regime. While PIAA can remove central obscurations, Ref. 41 presented a method (ACAD, Active Correction of Aperture Discontinuities) to derive mirror shapes suitable to remove the narrow structures introduced by spiders, gaps and maybe even missing segments. Because the required mirror deformations are relatively small (of the order of a micron), deformable mirrors (DMs) could be used for this purpose.

Even without apodization or remapping, PSF structures produced by gaps and spiders are typically less localized and less affected by the coronagraphic mask. Therefore, they show up mostly as bright structures of the original geometry in the Lyot plane of an efficient coronagraph and can be masked to a large extent by a suitable irregularly shaped Lyot stop. For larger separations the fraction of the field of view spoiled by spiders and gaps may be sufficiently small to ignore.

\subsection{Strut mitigation by apodizer optimization}

If two DMs are available, ACAD can be used to mitigate the diffraction effects due to the struts. However, if there is only one DM available, or no DMs at all, this task can be given to a different type of apodizer, specifically computed to take these additional diffraction effects into account. Following an idea first presented in Ref. 40, and then applied to the case of the four-quadrant phase mask, the $2 \mathrm{D}$ transmission of amplitude apodizers can 
be maximized in a numerical optimization problem, where constraints are set on the extremum values of the electric field in the Lyot plane.

An upcoming paper (A. Carlotti et al. 2013b, in preparation) presents charge-2 and charge-4 VC apodizers designed for several on-axis telescopes with 10-30\% central obscurations and orthogonal spiders. Interestingly, the overall morphology of these numerically optimized solutions converges to the analytical RAVC design for the ideal strut-less pupil, and departs from it only around the struts, where additional local apodization features are necessary. While slightly smaller, the transmissions of these apodized coronagraphs are also comparable to the transmissions of the RAVC2 and RAVC4. This is mostly due to the presence of the secondary supports in the pupil, but also partially explained by the finite radius imposed to the vortex phase mask (the representation of the vortex mask at the focal plane is currently limited to a radius of 32-64 $\lambda / D$ because of the complexity of the computations). As other 2D optimal apodizers, these masks have binary transmissions, and thus can also make use of the microdots and coating technologies, as discussed in Section 4.3.2.

Another straightforward strut mitigation technique is the spider removal plate (SRP) which removes the strut footprint by translating the clear and contiguous parts of the pupil inwards with tilted plane-parallel plates. ${ }^{42}$ This solution is however less ideal for very high contrast applications since it introduces a thick prismatic optical element in the beam upstream of the coronagraph, and with it, its share of chromatic optical aberrations.

\section{DISCUSSION AND CONCLUSIONS}

The two concepts presented here unambiguously solve the last hurdle that the VC faced, namely its sensitivity to central obscuration. The 3-stage MSVC is improving upon the 2-stage MSVC presented in Ref. 17, because it breaks the $\left(r_{0} / R\right)^{2 n}$ law to allow for infinite contrast (in the ideal case) for $n=3$. The MSVC builds on the multi-stage coronagraph heritage, which, despite its relative optical complexity, enables reduced sensitivity to the coronagraph chromaticity and tip-tilt errors. The RAVC on the other hand is a game-changing concept, because of its simplicity. It is also now extended to higher topological charges which are preferable when sensitivity to low-order aberrations and stellar diameters become an issue.

The two concepts are particularly relevant to future extreme adaptive optics instruments for Extremely Large Telescopes (ELT) and coronagraphic space missions employing on-axis telescopes. With a more limited aperture in space, the RAVC throughput loss might be an issue. However, a forthcoming paper (Pueyo et al. 2013, in preparation) will extend the RAVC concept to lossless apodization techniques, which should mitigate this problem as well.

\section{ACKNOWLEDGMENTS}

This work was carried out at the European Southern Observatory (ESO) site of Vitacura (Santiago, Chile).

\section{REFERENCES}

[1] Mawet, D., Riaud, P., Absil, O., and Surdej, J., "Annular Groove Phase Mask Coronagraph," ApJ 633, 1191-1200 (Nov. 2005).

[2] Mawet, D., Murakami, N., Delacroix, C., Serabyn, E., Absil, O., Baba, N., Baudrand, J., Boccaletti, A., Burruss, R., Chipman, R., Forsberg, P., Habraken, S., Hamaguchi, S., Hanot, C., Ise, A., Karlsson, M., Kern, B., Krist, J., Kuhnert, A., Levine, M., Liewer, K., McClain, S., McEldowney, S., Mennesson, B., Moody, D., Murakami, H., Niessner, A., Nishikawa, J., O’Brien, N., Oka, K., Park, P., Piron, P., Pueyo, L., Riaud, P., Sakamoto, M., Tamura, M., Trauger, J., Shemo, D., Surdej, J., Tabirian, N., Traub, W., Wallace, J., and Yokochi, K., "Taking the vector vortex coronagraph to the next level for ground- and space-based exoplanet imaging instruments: review of technology developments in the USA, Japan, and Europe," in [Society of Photo-Optical Instrumentation Engineers (SPIE) Conference Series], Society of Photo-Optical Instrumentation Engineers (SPIE) Conference Series 8151 (Sept. 2011). 
[3] Mawet, D., Pueyo, L., Lawson, P., Mugnier, L., Traub, W., Boccaletti, A., Trauger, J. T., Gladysz, S., Serabyn, E., Milli, J., Belikov, R., Kasper, M., Baudoz, P., Macintosh, B., Marois, C., Oppenheimer, B., Barrett, H., Beuzit, J.-L., Devaney, N., Girard, J., Guyon, O., Krist, J., Mennesson, B., Mouillet, D., Murakami, N., Poyneer, L., Savransky, D., Vérinaud, C., and Wallace, J. K., "Review of small-angle coronagraphic techniques in the wake of ground-based second-generation adaptive optics systems," in [Proc. SPIE], 8442 (Sept. 2012).

[4] Mawet, D., Serabyn, E., Liewer, K., Burruss, R., Hickey, J., and Shemo, D., "The Vector Vortex Coronagraph: Laboratory Results and First Light at Palomar Observatory," ApJ 709, 53-57 (Jan. 2010).

[5] Mawet, D., Mennesson, B., Serabyn, E., Stapelfeldt, K., and Absil, O., "A Dim Candidate Companion to epsilon Cephei," ApJl 738, L12 (Sept. 2011).

[6] Serabyn, E., Mawet, D., and Burruss, R., "An image of an exoplanet separated by two diffraction beamwidths from a star," Nature 464, 1018-1020 (Apr. 2010).

[7] Wahl, M., Metchev, S. A., Patel, R., Serabyn, G., and PALM-3000 Adaptive Optics Team, "Extreme Contrast Direct Imaging of Planets and Debris disks with the Palomar P3K Adaptive Optics System and the Vector Vortex Coronagraph," in [American Astronomical Society Meeting Abstracts], American Astronomical Society Meeting Abstracts 221, 144.22 (Jan. 2013).

[8] Mawet, D., Absil, O., Delacroix, C., Girard, J. H., Milli, J., O’Neal, J., Baudoz, P., Boccaletti, A., Bourget, P., Christiaens, V., Forsberg, P., Gonte, F., Habraken, S., Hanot, C., Karlsson, M., Kasper, M., Lizon, J.-L., Muzic, K., Olivier, R., Peña, E., Slusarenko, N., Tacconi-Garman, L. E., and Surdej, J., "L'-band AGPM vector vortex coronagraph's first light on VLT/NACO. Discovery of a late-type companion at two beamwidths from an F0V star," A $\& A$ 552, L13 (Apr. 2013).

[9] Martinache, F., Guyon, O., Clergeon, C., Garrel, V., and Blain, C., "The Subaru coronagraphic extreme AO project: first observations," in [Proc. SPIE], 8447 (July 2012).

[10] Skrutskie, M. F., Jones, T., Hinz, P., Garnavich, P., Wilson, J., Nelson, M., Solheid, E., Durney, O., Hoffmann, W., Vaitheeswaran, V., McMahon, T., Leisenring, J., and Wong, A., "The Large Binocular Telescope mid-infrared camera (LMIRcam): final design and status," in [Proc. SPIE], 7735 (July 2010).

[11] Esposito, S., Riccardi, A., Pinna, E., Puglisi, A., Quirós-Pacheco, F., Arcidiacono, C., Xompero, M., Briguglio, R., Agapito, G., Busoni, L., Fini, L., Argomedo, J., Gherardi, A., Brusa, G., Miller, D., Guerra, J. C., Stefanini, P., and Salinari, P., "Large Binocular Telescope Adaptive Optics System: new achievements and perspectives in adaptive optics," in [Proc. SPIE], 8149 (Sept. 2011).

[12] Spergel, D., Gehrels, N., Breckinridge, J., Donahue, M., Dressler, A., Gaudi, B. S., Greene, T., Guyon, O., Hirata, C., Kalirai, J., Kasdin, N. J., Moos, W., Perlmutter, S., Postman, M., Rauscher, B., Rhodes, J., Wang, Y., Weinberg, D., Centrella, J., Traub, W., Baltay, C., Colbert, J., Bennett, D., Kiessling, A., Macintosh, B., Merten, J., Mortonson, M., Penny, M., Rozo, E., Savransky, D., Stapelfeldt, K., Zu, Y., Baker, C., Cheng, E., Content, D., Dooley, J., Foote, M., Goullioud, R., Grady, K., Jackson, C., Kruk, J., Levine, M., Melton, M., Peddie, C., Ruffa, J., and Shaklan, S., "WFIRST-2.4: What Every Astronomer Should Know," ArXiv e-prints (May 2013).

[13] Spergel, D., Gehrels, N., Breckinridge, J., Donahue, M., Dressler, A., Gaudi, B. S., Greene, T., Guyon, O., Hirata, C., Kalirai, J., Kasdin, N. J., Moos, W., Perlmutter, S., Postman, M., Rauscher, B., Rhodes, J., Wang, Y., Weinberg, D., Centrella, J., Traub, W., Baltay, C., Colbert, J., Bennett, D., Kiessling, A., Macintosh, B., Merten, J., Mortonson, M., Penny, M., Rozo, E., Savransky, D., Stapelfeldt, K., Zu, Y., Baker, C., Cheng, E., Content, D., Dooley, J., Foote, M., Goullioud, R., Grady, K., Jackson, C., Kruk, J., Levine, M., Melton, M., Peddie, C., Ruffa, J., and Shaklan, S., "Wide-Field InfraRed Survey TelescopeAstrophysics Focused Telescope Assets WFIRST-AFTA Final Report," ArXiv e-prints (May 2013).

[14] Trauger, J., Stapelfeldt, K., Traub, W., Krist, J., Moody, D., Mawet, D., Serabyn, E., Henry, C., Brugarolas, P., Alexander, J., Gappinger, R., Dawson, O., Mireles, V., Park, P., Pueyo, L., Shaklan, S., Guyon, O., Kasdin, J., Vanderbei, R., Spergel, D., Belikov, R., Marcy, G., Brown, R. A., Schneider, J., Woodgate, B., Egerman, R., Matthews, G., Elias, J., Conturie, Y., Vallone, P., Voyer, P., Polidan, R., Lillie, C., Spittler, C., Lee, D., Hejal, R., Bronowicki, A., Saldivar, N., Ealey, M., and Price, T., "ACCESS: a concept study for the direct imaging and spectroscopy of exoplanetary systems," in [Society of PhotoOptical Instrumentation Engineers (SPIE) Conference Series], Society of Photo-Optical Instrumentation Engineers (SPIE) Conference Series $\mathbf{7 7 3 1}$ (July 2010). 
[15] Boccaletti, A., Schneider, J., Traub, W., Lagage, P.-O., Stam, D., Gratton, R., Trauger, J., Cahoy, K., Snik, F., Baudoz, P., Galicher, R., Reess, J.-M., Mawet, D., Augereau, J.-C., Patience, J., Kuchner, M., Wyatt, M., Pantin, E., Maire, A.-L., Vérinaud, C., Ronayette, S., Dubreuil, D., Min, M., Rodenhuis, M., Mesa, D., Belikov, R., Guyon, O., Tamura, M., Murakami, N., and Beerer, I. M., "SPICES: spectropolarimetric imaging and characterization of exoplanetary systems. From planetary disks to nearby Super Earths," Experimental Astronomy 34, 355-384 (Oct. 2012).

[16] Mawet, D., Pueyo, L., Moody, D., Krist, J., and Serabyn, E., "The Vector Vortex Coronagraph: sensitivity to central obscuration, low-order aberrations, chromaticism, and polarization," in [Society of Photo-Optical Instrumentation Engineers (SPIE) Conference Series], Society of Photo-Optical Instrumentation Engineers (SPIE) Conference Series 7739 (July 2010).

[17] Mawet, D., Serabyn, E., Wallace, J. K., and Pueyo, L., "Improved high-contrast imaging with on-axis telescopes using a multistage vortex coronagraph," Optics Letters 36, 1506 (Apr. 2011).

[18] Jenkins, C., "Optical vortex coronagraphs on ground-based telescopes," MNRAS 384, 515-524 (Feb. 2008).

[19] Swartzlander, Jr., G. A., "The optical vortex coronagraph," Journal of Optics A: Pure and Applied Optics 11, 094022 (Sept. 2009).

[20] Carlotti, A., Ricort, G., and Aime, C., "Phase mask coronagraphy using a Mach-Zehnder interferometer," AEAA 504, 663-671 (Sept. 2009).

[21] Yaitskova, N., "Double stage Lyot coronagraph with the apodized reticulated stop for the Extremely Large Telescope," in [Society of Photo-Optical Instrumentation Engineers (SPIE) Conference Series], Coulter, D. R., ed., Society of Photo-Optical Instrumentation Engineers (SPIE) Conference Series 5905, 292-302 (Aug. 2005).

[22] Baudoz, P., Assemat, F., Galicher, R., Baudrand, J., and Boccaletti, A., "Experimental results of multi-stage four quadrant phase mask coronagraph," in [Society of Photo-Optical Instrumentation Engineers (SPIE) Conference Series], Society of Photo-Optical Instrumentation Engineers (SPIE) Conference Series 7735 (July 2010).

[23] Galicher, R., Baudoz, P., and Baudrand, J., "Multi-stage four-quadrant phase mask: achromatic coronagraph for space-based and ground-based telescopes," AËA 530, A43 (June 2011).

[24] Soummer, R., "Apodized Pupil Lyot Coronagraphs for Arbitrary Telescope Apertures," ApJl 618, L161L164 (Jan. 2005).

[25] Guyon, O., Hinz, P. H., Cady, E., Belikov, R., and Martinache, F., "High Performance Lyot and PIAA Coronagraphy for Arbitrarily shaped Telescope Apertures," ArXiv e-prints (May 2013).

[26] Mawet, D., Serabyn, E., Liewer, K., Hanot, C., McEldowney, S., Shemo, D., and O'Brien, N., "Optical Vectorial Vortex Coronagraphs using Liquid Crystal Polymers: theory, manufacturing and laboratory demonstration," Optics Express 17, 1902-1918 (Feb. 2009).

[27] Errmann, R., Minardi, S., and Pertsch, T., "A Broadband Scalar Vortex Coronagraph," ArXiv e-prints (July 2013).

[28] Delacroix, C., Absil, O., Forsberg, P., Mawet, D., Christiaens, V., Karlsson, M., Boccaletti, A., Baudoz, P., Kuittinen, M., Vartiainen, I., Surdej, J., and Habraken, S., "Laboratory demonstration of a mid-infrared AGPM vector vortex coronagraph," A 8 A 553, A98 (May 2013).

[29] Murakami, N., Nishikawa, J., Yokochi, K., Tamura, M., Baba, N., and Abe, L., "Achromatic Eight-octant Phase-mask Coronagraph using Photonic Crystal," ApJ 714, 772-777 (May 2010).

[30] Murakami, N., Hamaguchi, S., Sakamoto, M., Fukumoto, R., Ise, A., Oka, K., Baba, N., and Tamura, M., "Design and laboratory demonstration of an achromatic vector vortex coronagraph," Optics Express 21, 7400 (Mar. 2013).

[31] Soummer, R., Sivaramakrishnan, A., Pueyo, L., Macintosh, B., and Oppenheimer, B. R., "Apodized Pupil Lyot Coronagraphs for Arbitrary Apertures. III. Quasi-achromatic Solutions," ApJ 729, 144 (Mar. 2011).

[32] Kasper, M., Beuzit, J.-L., Feldt, M., Dohlen, K., Mouillet, D., Puget, P., Wildi, F., Abe, L., Baruffolo, A., Baudoz, P., Bazzon, A., Boccaletti, A., Brast, R., Buey, T., Chesneau, O., Claudi, R., Costille, A., Delboulbé, A., Desidera, S., Dominik, C., Dorn, R., Downing, M., Feautrier, P., Fedrigo, E., Fusco, T., Girard, J., Giro, E., Gluck, L., Gonte, F., Gojak, D., Gratton, R., Henning, T., Hubin, N., Lagrange, A.M., Langlois, M., Mignant, D. L., Lizon, J.-L., Lilley, P., Madec, F., Magnard, Y., Martinez, P., Mawet, D., 
Mesa, D., Müller-Nilsson, O., Moulin, T., Moutou, C., O’Neal, J., Pavlov, A., Perret, D., Petit, C., Popovic, D., Pragt, J., Rabou, P., Rochat, S., Roelfsema, R., Salasnich, B., Sauvage, J.-F., Schmid, H. M., Schuhler, N., Sevin, A., Siebenmorgen, R., Soenke, C., Stadler, E., Suarez, M., Turatto, M., Udry, S., Vigan, A., and Zins, G., "Gearing up the SPHERE," The Messenger 149, 17-21 (Sept. 2012).

[33] Macintosh, B. A., Graham, J. R., Palmer, D. W., Doyon, R., Dunn, J., Gavel, D. T., Larkin, J., Oppenheimer, B., Saddlemyer, L., Sivaramakrishnan, A., Wallace, J. K., Bauman, B., Erickson, D. A., Marois, C., Poyneer, L. A., and Soummer, R., "The Gemini Planet Imager: from science to design to construction," Adaptive Optics Systems. Edited by Hubin 7015, 31 (July 2008).

[34] Martinez, P., Dorrer, C., Aller Carpentier, E., Kasper, M., Boccaletti, A., Dohlen, K., and Yaitskova, N., "Design, analysis, and testing of a microdot apodizer for the Apodized Pupil Lyot Coronagraph," A\&SA 495, 363-370 (Feb. 2009).

[35] Martinez, P., Dorrer, C., Kasper, M., Boccaletti, A., and Dohlen, K., "Design, analysis, and testing of a microdot apodizer for the apodized pupil Lyot coronagraph. II. Impact of the dot size," A\&A A 500, 1281-1285 (June 2009).

[36] Krist, J. E., Balasubramanian, K., Beichman, C. A., Echternach, P. M., Green, J. J., Liewer, K. M., Muller, R. E., Serabyn, E., Shaklan, S. B., Trauger, J. T., Wilson, D. W., Horner, S. D., Mao, Y., Somerstein, S. F., Vasudevan, G., Kelly, D. M., and Rieke, M. J., "The JWST/NIRCam coronagraph: mask design and fabrication," in [Society of Photo-Optical Instrumentation Engineers (SPIE) Conference Series], Society of Photo-Optical Instrumentation Engineers (SPIE) Conference Series 7440 (Aug. 2009).

[37] Trauger, J., Moody, D., Gordon, B., Krist, J., and Mawet, D., "Complex apodization Lyot coronagraphy for the direct imaging of exoplanet systems: design, fabrication, and laboratory demonstration," in [Society of Photo-Optical Instrumentation Engineers (SPIE) Conference Series], Society of Photo-Optical Instrumentation Engineers (SPIE) Conference Series 8442 (Sept. 2012).

[38] Trauger, J., Moody, D., Gordon, B., Krist, J., and Mawet, D., "A hybrid Lyot coronagraph for the direct imaging and spectroscopy of exoplanet systems: recent results and prospects," in [Society of Photo-Optical Instrumentation Engineers (SPIE) Conference Series], Society of Photo-Optical Instrumentation Engineers (SPIE) Conference Series $\mathbf{8 1 5 1}$ (Sept. 2011).

[39] Carlotti, A., Vanderbei, R., and Kasdin, N. J., "Optimal pupil apodizations of arbitrary apertures for high-contrast imaging," Optics Express 19, 26796 (Dec. 2011).

[40] Carlotti, A., "Apodized phase mask coronagraphs for arbitrary apertures," $A \& A$ 551, A10 (Mar. 2013).

[41] Pueyo, L. and Norman, C., "High Contrast Imaging with an Arbitrary Aperture: Active Correction of Aperture Discontinuities," ArXiv e-prints (Nov. 2012).

[42] Lozi, J., Martinache, F., and Guyon, O., "Phase-Induced Amplitude Apodization on Centrally Obscured Pupils: Design and First Laboratory Demonstration for the Subaru Telescope Pupil," PASP 121, 1232-1244 (Nov. 2009). 